\title{
Erchen Decoction Ameliorates Lipid Metabolism by the Regulation of the Protein CAV-1 and the Receptors VLDLR, LDLR, ABCA1, and SRB1 in a High-Fat Diet Rat Model
}

\author{
Shanshan Ding $\mathbb{D},{ }^{1,2}$ Jie Kang $\mathbb{D},,^{1,2}$ Ling Tong $\mathbb{D},{ }^{1}$ Yuchen Lin $\mathbb{D}{ }^{1}$ \\ Linghong Liao, ${ }^{1,2}$ and Bizhen Gao ${ }^{2}$ \\ ${ }^{1}$ College of Traditional Chinese Medicine, Fujian University of Traditional Chinese Medicine, Fuzhou 350122, China \\ ${ }^{2}$ Fujian Key Laboratory of TCM Health State, Fujian University of Traditional Chinese Medicine, Fuzhou 350122, China
}

Correspondence should be addressed to Shanshan Ding; ding123shan@163.com

Received 22 March 2018; Revised 7 September 2018; Accepted 24 September 2018; Published 8 October 2018

Academic Editor: Massimo Nabissi

Copyright (c) 2018 Shanshan Ding et al. This is an open access article distributed under the Creative Commons Attribution License, which permits unrestricted use, distribution, and reproduction in any medium, provided the original work is properly cited.

Lipid metabolism disorder is a common metabolic disorder characterized by abnormal lipid levels in blood. Erchen decoction (ECD) is a traditional Chinese medicine prescription, which is used for the treatment of diseases caused by retention of phlegm dampness. It has been reported to ameliorate the disorder of lipid metabolism. The aim of the present study was to investigate the effects and underlying mechanisms of ECD in lipid metabolism disorder induced by a high-fat diet (HFD) in rats. ECD (4.35g/kg/d) and atorvastatin $(10 \mathrm{mg} / \mathrm{kg} / \mathrm{d}$, positive control) were orally administered to HFD-fed rats for four weeks. The parameters, food, water consumption, body weight, body length, liver, and visceral fat weight and the content of serum lipids and lipid transporters were assessed. The effects of ECD on the mRNA and protein expression levels of lipid transport factors were measured by real-time PCR and western blotting. The present study demonstrated that ECD improved the disorders of serum lipid and lipid transporters in HFD-fed rats, TG $(0.70 \pm 0.08 \mathrm{mmol} / \mathrm{L}, p<0.01)$, LDL-C $(1.50 \pm 0.19 \mathrm{mmol} / \mathrm{L}, p<0.01), \mathrm{LDL}(1.38 \pm 0.21 \mathrm{mmol} / \mathrm{L}, p<0.05)$, and oxLDL $(1.77 \pm 0.39 \mathrm{ng} / \mathrm{mL}, p<0.05)$ were downregulated, while HDL-C $(0.87 \pm 0.13 \mathrm{mmol} / \mathrm{L}, p<0.01), \mathrm{FFA}(0.62 \pm 0.13 \mathrm{mmol} / \mathrm{L}, p<0.05), \mathrm{HDL}$ $(38.8 \pm 4.0 \mathrm{mg} / \mathrm{dL}, p<0.05)$, and CETP $(903.6 \pm 120.0 \mathrm{ng} / \mathrm{mL}, p<0.05)$ were upregulated. But ECD obviously had no effects on the indices food/water/energy intake, body/tissue (liver and fat) weight, and BMI ( $p>0.05$ ). Concomitantly, ECD reversed the abnormal expressions of those lipid transport factors in the liver and visceral fat.

\section{Introduction}

Lipid metabolism disorder refers to a panel of diseases that are characterized by the disruption of lipid metabolism, such as LDL-hypercholesterolemia, hypertriglyceridemia, mixed hyperlipoproteinemia, and low HDL cholesterol [1]. These disorders are accompanied by abnormal lipid biochemical indices that are usually elevated and include exceeding serum levels of total cholesterol (TC), triglyceride (TG), low density lipoprotein cholesterol (LDL-C), and/or lower level of high density lipoprotein cholesterol (HDL-C) [2]. Lipid metabolism disorder is one of the major risk factors of fatty liver, diabetes, coronary heart disease, atherosclerosis, hypertension, and various cardiovascular diseases [3]. The LDL cholesterol has been recommended as the predominant marker for the early prognosis of cardiovascular diseases [1]. The current European recommendations state that an LDLcholesterol target value is defined on the basis of the overall cardiovascular risk [1]. The decline in the LDL-cholesterol concentration with the use of statins is considered the most effective type of pharmacotherapy. Moreover, atorvastatin (ATV) has been preferred over other satins initially due to its effective hypolipidemic effect. So it is widely used as a positive control to lower elevated lipid levels; in studies the dose of ATV for rats ranges from 2.08 to $20 \mathrm{mg} / \mathrm{kg} /$ day, but the most commonly used effective dose is $10 \mathrm{mg} / \mathrm{kg} / \mathrm{day}$ [4-6]. However, statins cannot be applied to all populations worldwide due to side effects, whereas in certain cases their efficacy in lowering cholesterol level is not adequate [7]. About $20 \%$ of patients treated with this medication suffer from statin related 
myalgia, with long-term therapy; it may extends from mild myopathy to fatal rhabdomyolysis. Another impediment with statin therapy is hepatotoxicity, characterized by an elevation of serum transaminases. It has been observed in $1-3 \%$ of the individuals treated with atorvastatin and it is predicted that prolonged use of atorvastatin at high doses may lead to rare events of autoimmune hepatitis and deaths [8-10]. Consequently, alternative treatments for lipid metabolism disorders are required.

According to traditional Chinese medicine (TCM), lipid metabolism disorder belongs to the category of phlegm dampness which is formed by the poor transportation of nutrients [11-13]. Erchen decoction (ECD) is a fundamental TCM prescription for the treatment of drying dampness and resolving phlegm [14]. The major components of each herbal medicine in ECD are known to be bioactive components, including flavonoids (e.g., liquiritin, liquiritigenin, hesperidin, rutin, naringin, neohesperidin, and poncirin), triterpenoids (e.g., glycyrrhizin, pachymic acid, and eburicoic acid), and phenolic acids (e.g., homogentisic acid) [15]. With regard to the clinical application of TCM, ECD is extensively used for the treatment of a variety of diseases caused by retention of phlegm dampness, such as obesity, fatty liver, diabetes, and hypertension [16]. Previous studies conducted by our research group and other research groups [17-20] have shown that ECD could improve lipid metabolic disorder. In addition, ECD can regulate glucose and lipid metabolism, reduce lipid disposition, alleviate insulin resistance, and suppress inflammation [21, 22]. ECD, as a Chinese herbal medicine, apply a multicomponent and multitarget approach in the treatment of diseases, so it has definite therapeutic effect with little side effects and wide indications. ECD may be more appropriate for patients with adverse reactions to atorvastatin, as mentioned above. Despite multiple treatment effects observed, the molecular mechanism of ECD in the regulation of metabolic disorder is still unclear.

Lipid metabolism disorder is a direct result of abnormal lipid transport, via lipid transporters and their receptors that play key roles in lipid transport. Caveolae are 50-100 nm stable lipid raft regions abundant in vascular endothelial cells, adipocytes, and fibroblasts [23]. These organelles play key roles in endocytosis and cholesterol homeostasis and are composed of a cholesterol and sphingolipid rich environment that binds to certain receptors, such as gp130 [24-26]. Caveolin-1-3 proteins are responsible for the formation and function of caveolae. Caveolin-1(CAV-1) has been reported to correlate well with lipid metabolism [27]. Caveolae mediate transmembrane lipid transportation and the endocytosis and transcytosis of lipoprotein molecules [28, 29]. Caveolae in the plasma membrane may invaginate and form caveolar vesicles that contain lipids or lipoproteins. With the exception of vesicle-mediated lipid transportation, various receptors in caveolae, such as LDLR, SR-B1, and ABC-A1, mediate lipid efflux [27]. CAV-1 is enriched in caveolae, endoplasmic reticulum, and Golgi bodies and shuttles between the cytoplasm and the cell membrane via the intracellular trafficking of caveolar vesicles $[30,31]$. The lipoprotein receptors, such as the very low density lipoprotein receptor (VLDLR) and the low density lipoprotein receptor (LDLR), and the lipid transporters, such as the ATP-binding cassette transporterA1 (ABCA1) and the scavenger receptor class B type I (SRB1) comprise a transmembrane lipid transport system [32-35]. Lipoproteins, including very low density lipoprotein (VLDL), low density lipoprotein (LDL), high density lipoprotein (HDL), and the protein cholesteryl ester transfer protein (CETP) constitute an extracellular lipid trafficking system [36]. The modulation of the levels of lipoprotein receptors by lipid lowering drugs is a mechanism of action that contributes to the decrease of cholesterol and triglycerides in the serum. Despite the potential clinical benefit of ECD in preventing high-fat diet-induced metabolic disorders, the exact mode of action remains poorly defined. In the present study, we aimed to explore the underlying mechanism of ECD lipid lowering action by investigating its effect on the lipid transport system, as determined in caveolae.

\section{Materials and Methods}

2.1. Diet and Drug Preparation. The high-fat diet (HFD) contained $60 \%$ (cal) fat, $20 \%$ (cal) carbohydrates, and $20 \%$ (cal) protein with total calorific value of $5.24 \mathrm{kcal} / \mathrm{g}$ (D12492, Research Diets, New Brunswick, NJ, USA). The normal diet (NFD) contained 12.1\% (cal) fat, 22.5\% (cal) protein, and $65.4 \%$ (cal) carbohydrate with total calorific value of $3.42 \mathrm{kcal} / \mathrm{g}$ (Beijing Huafukang Bioscience Co. Inc., Beijing, China).

According to the textbook of The Taiping Huimin Heji Jufang, Erchen decoction (ECD) comprises four Chinese herbs: Rhizoma Pinelliae (15g), Pericarpium Citri Reticulatae (15g), Poria (9g), and Radix Glycyrrhizae (4.5g). These herbs were purchased from Guoyi Hospital that was affiliated to Fujian University of TCM. ECD was prepared according to the conventional TCM decocting method [1]. All herbs were placed in a cooking pot (porcelain) with $500 \mathrm{~mL}$ of water and were soaked for $30 \mathrm{~min}$. Subsequently, the herbs were boiled and simmered for $20 \mathrm{~min}$. The liquid was transferred by filtration and water was added to boil the remaining herbs. The process was repeated one more time and the two preparations were mixed to produce a total volume of $250 \mathrm{~mL}$ in a glass jar. The mixture was concentrated to $100 \mathrm{~mL}$ by a rotary evaporator. The doses of ECD we used in the current study were approximately 6 times higher than the standard dose used in clinical practice, according to the dose-equivalence equation between rats and humans [37]. ECD $(4.35 \mathrm{~g} / \mathrm{kg}$ ) and atorvastatin (ATV, $10 \mathrm{mg} / \mathrm{kg}$ ) were administered at a dose of $10 \mathrm{~mL} / \mathrm{kg} / \mathrm{d}$ (pure solution).

2.2. Animals and Experimental Design. Adult male Sprague Dawley rats weighing 180-200 g were obtained from Shanghai Slac Laboratory Animal Company (Shanghai, China). All rats had free access to water and food under standard laboratory conditions (temperature of $24 \pm 2^{\circ} \mathrm{C}$; humidity of $55 \pm 10 \%$; 12-hour light/dark cycle, light on at 08:00). The experimental protocol was approved by the Fujian University of TCM Ethics Committee for the use of experimental animals (SCXK 2014-004). Following one week of acclimatization $(0 \mathrm{w})$, the rats were randomly divided into four groups: NFD ( $n=14)$, HFD ( $n=14)$, ECD (HFD-fed rats treated with 
TABLE 1: Primer sequences used for real-time PCR analysis.

\begin{tabular}{lcc}
\hline Gene & Forward primer & Reverse primer \\
\hline CAV-1 & TAAACCCATTCCTGCTCTCC & CGCCTCCCAGTCTTCCTATT \\
VLDLR & CACCAAAGTATGTGACCAGGAA & AACAGCCACCGTTATTGACC \\
LDLR & TAGGGTTTCTGCTCTTCACCA & GCCACCACATTCTTCAGGTT \\
SRB1 & GGACAAGCAGTTCCAGATCC & GCCAATTCAGTGTTCAGGTG \\
ABCA1 & TGCTTCCGTTATCCAACTCC & TCCCTAATGCTGGTGTCCTT \\
$\beta$-Actin & CACCCGCGAGTACAACCTTC & CCCATACCCACCATCACACC \\
\hline
\end{tabular}

Erchen decoction, $\mathrm{n}=14$ ), and ATV (HFD-fed rats treated with atorvastatin, $n=14$ ). The rats received a HFD for 8 weeks (1-8w) prior the administration of ECD and/or ATV in order to establish a lipid metabolism disorder model. During this period, the success of model establishment was assessed by the measurement of the change in blood lipid levels [3840]. ECD and ATV were administered to the corresponding animal groups, whereas saline was administered to the NFD and HFD groups by gastric gavage for a period of 4 weeks (9-12w). During the compound administration, HFD-fed rats maintained a high-fat diet in order to prevent self-healing. All rats could eat and drink ad libitum. Food and water consumption were recorded daily, whereas body weight and length were measured weekly. Estimation of energy intake was done indirectly by using the formula: Energy intake = food consumption $\times$ food total calorific value $(5.24 \mathrm{kcal} / \mathrm{g}$ or $3.42 \mathrm{kcal} / \mathrm{g}$ ). Following 4 weeks of drug treatment, the rats were anesthetized with pentobarbital ( $40 \mathrm{mg} / \mathrm{kg}$ body weight) and blood was collected via the abdominal aorta. Their tissues were removed, weighted, and stored at $-80^{\circ} \mathrm{C}$.

2.3. Serum Lipids Content Measurements. Blood was collected from the abdominal aorta into a tube and was centrifuged at $3,500 \mathrm{rpm}$ for $15 \mathrm{~min}$ at $4^{\circ} \mathrm{C}$. The serum was transferred into a separate vial and stored at $4^{\circ} \mathrm{C}$. The concentration of serum triglyceride (TG), total cholesterol (TC), high density lipoprotein cholesterol (HDL-C), low density lipoprotein cholesterol (LDL-C), and free fatty acids (FFAs) were measured using biochemical assay kit according to the manufacturer's instruction (Nanjing Jiancheng Bioengineering Institute, Nanjing, China).

2.4. Serum Lipid Transporters Assay. Serum lipid transporters, VLDL, LDL, oxLDL, HDL, and CETP, were measured using the enzyme linked immunosorbent assay kit according to the manufacturer's instruction (Shanghai Meilian Biological Technology Co., Ltd., Shanghai, China).

2.5. Real-Time PCR. Total RNA was extracted from liver tissues and visceral fat using RNAiso plus (TaKaRa, Otsu, Japan). Complementary DNA (cDNA) was synthesized using random primers and reverse transcriptase as recommended by the manufacturer instructions (TaKaRa, Otsu, Japan). To evaluate the mRNA expression of lipid transport factors that were derived from caveolae in liver tissues and visceral fat, real-time PCR was conducted using a SYBR Green PCR master mix (TaKaRa, Otsu, Japan) according to the manufacturer's instructions. The reactions were carried out in the Mastercycler ep realplex 4S real-time PCR system (Eppendorf, Hamburg, Germany). The sequences of the primers are described in Table 1 . The cDNA was denatured at $95^{\circ} \mathrm{C}$ for 30 s followed by 40 cycles of PCR that included a denaturation step at $95^{\circ} \mathrm{C}$ for $5 \mathrm{~s}$ and an annealing step at $60^{\circ} \mathrm{C}$ for $30 \mathrm{~s}$. The mRNA levels of the target genes were normalized to the mRNA levels of the $\beta$-actin gene and the results were expressed as fold changes of the threshold cycle $(\mathrm{Ct})$ value relative to the control samples using the $2^{-\Delta \Delta \mathrm{Ct}}$ method [41].

2.6. Western Blotting. The liver tissue and visceral fat of each group were homogenized in liquid nitrogen, and wholecell protein was extracted using lysate buffer that contained proteinase inhibitors (MDL biotech Co., Ltd., Beijing, China). The protein concentration was quantified spectrophotometrically using the BSA protein assay kit (MDL biotech Co., Ltd., Beijing, China). Equal protein amounts were separated by sodium dodecyl sulfate polyacrylamide gel electrophoresis and transferred on polyvinylidene difluoride membranes, which were subsequently blocked with $1 \%$ bovine serum albumin and incubated with primary antibodies against CAV-1 (1:500, Cell Signaling Technology, Beverly, MA, USA), VLDLR (1:200, Abcam, Cambridge, UK), LDLR (1:200, Abcam, Cambridge, UK), SR-B1 (1:200, Abcam, Cambridge, UK), ABCA1 (1:200, Abcam, Cambridge, UK), and $\beta$-actin (MDL biotech Co., Ltd., Beijing, China) overnight at $4^{\circ} \mathrm{C}$. Following washing of the membranes with PBS-Tween, the blots were incubated with HRP-conjugated secondary antibodies (1:3,000, Goat Anti-Mouse/Rabbit IgG; MDL biotech Co., Ltd., Beijing, China) for $60 \mathrm{~min}$ at room temperature. The protein bands were detected using enhanced chemiluminescence (ECL; MDL biotech Co., Ltd., Beijing, China) and finally scanned by the ChemiDoc MP system (BioRad, USA). The data were quantified by densitometry and presented as the ratio of the target protein levels to the levels of $\beta$-actin.

2.7. Statistical Analysis. Data analyses were performed using the statistical program SPSS 19.0 (SPSS Inc., Chicago, IL, USA). All data were expressed as mean \pm SE. Significant differences among groups were evaluated using one-way ANOVA followed by the Tukey tests for post hoc, where equal variances were assumed and/or followed by Tamhane's T2 tests where equal variances were not assumed. The values for the food, water and energy intake, body weight, BMI, and serum lipid were compared by repeated measures ANOVA 

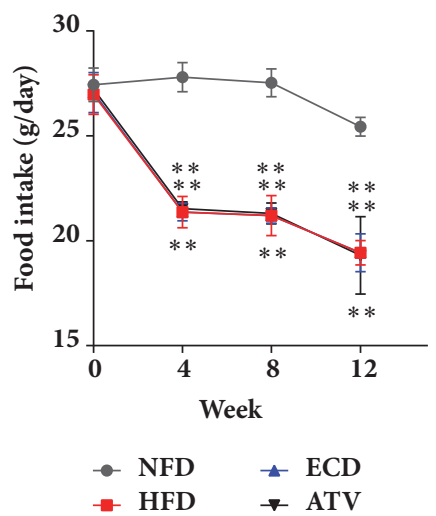

(a)

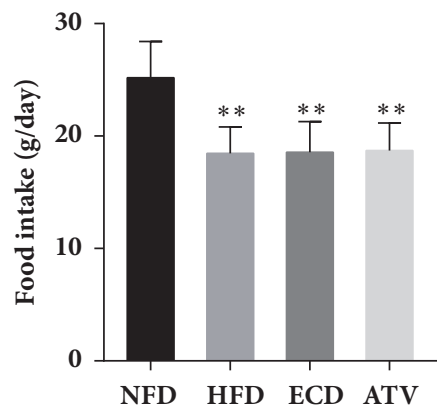

(d)

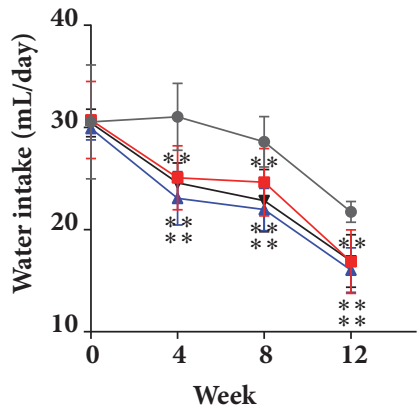

$\rightarrow$ NFD $\rightarrow$ ECD

(b)

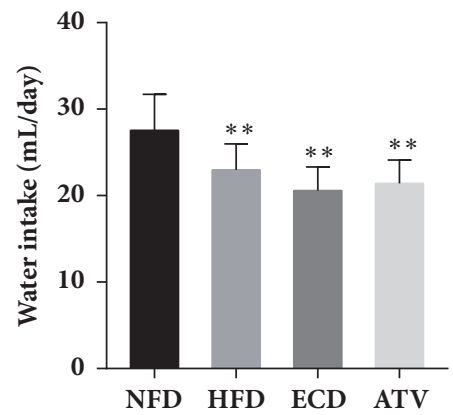

(e)

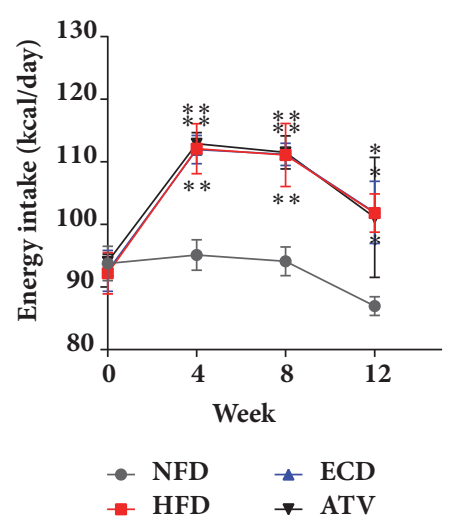

(c)

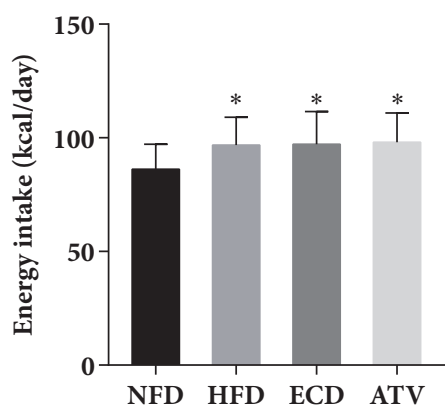

(f)

FIGURE 1: Measurements of daily food, water, and energy intake in the NFD, HFD, ECD, and ATV groups. (a) Food intake, (b) water intake, and (c) energy intake (weeks 0-4-8-12). (d) Food intake, (e) water intake, and (f) energy intake (week 12). The data are presented as mean \pm SEM $(\mathrm{n}=14), * p<0.05$ and $* * p<0.01$ versus NFD.

with a $4 \times 4$ (group $\times$ time). If the results of the RMANOVA were significant, paired t-tests using a Bonferroni adjustment were conducted as a post hoc analysis. The differences were considered significant for a $p$ value less than $0.05(p<0.05)$ and nonsignificant for a $p$ value higher than 0.05 ( $p>0.05)$.

\section{Results}

3.1. ECD and ATV Do Not Affect Food, Water, and Energy Intake in HFD Rats. Before HFD, the parameters daily food, water, and energy intake were not different among the 4 groups of animals, namely, NFD, HFD, ECD, and ATV. Food and water intake were significantly decreased in HFD, ECD, and ATV groups compared with the NFD group following feeding the HFD for four weeks; the effects were maintained until the $12^{\text {th }}$ week (Figures 1(a), 1(b), 1(d), and 1(e)). The opposite effect was noted for the parameter energy intake with regard to the comparison of the 3 groups with the NFD group (Figures 1(c) and 1(f)). No significant differences were observed among the 3 groups with regard to the aforementioned parameters (Figure 1).

3.2. The Effects of ECD on the Body Weight, Body Mass Index and Weight of Liver, Perirenal Fat, and Epididymis Fat Tissues. On the first day of experiment, the body weight and body mass index (BMI) in each group were not significantly different. Following feeding of the rats with HFD for four weeks, the animals of the groups HFD, ECD, and ATV exhibited significantly higher body weights and body mass indices (BMI) compared with the NFD group (Figures 2(a) and $2(\mathrm{~b}))$. The effects were maintained until the $8^{\text {th }}$ week. After the medication intervention, the body weight in the groups ECD and ATV was still markedly higher than in the NFD group, while the BMI were not significantly different with the NFD group (Figure 2).

In addition to the body mass index and animal weight, the weights of the liver, perirenal fat, and epididymis fat tissues were measured on week 12. Compared with the NFD group, the percentage of perirenal fat $(\mathrm{PF})$ and epididymis fat $(\mathrm{EF})$ tissue weight to body weight was considerably increased in the groups HDF, ECD, and ATV. However, with regard to the liver tissue, no significant differences were noted for the parameter tissue weight/body weight among the 4 groups (Figure 3).

3.3. ECD Affects the Levels of TG, TC, HDL-C, LDL-C, and FFAs in HFD Rats. The lipid markers sera TG, TC, HDL-C, LDL-C, and FFAs were measured in the 4 groups (Figure 4). Sera TG, TC, and LDL-C were significantly increased in the HFD group compared with the NFD group, while HDL$\mathrm{C}$ and FFAs were decreased significantly (Figure 4). ECD and ATV supplementation significantly alleviated the HFDinduced disorder in the markers TG, TC, HDL-C, LDL-C, and FFAs(Figure 4). ECD and ATV decreased significantly 


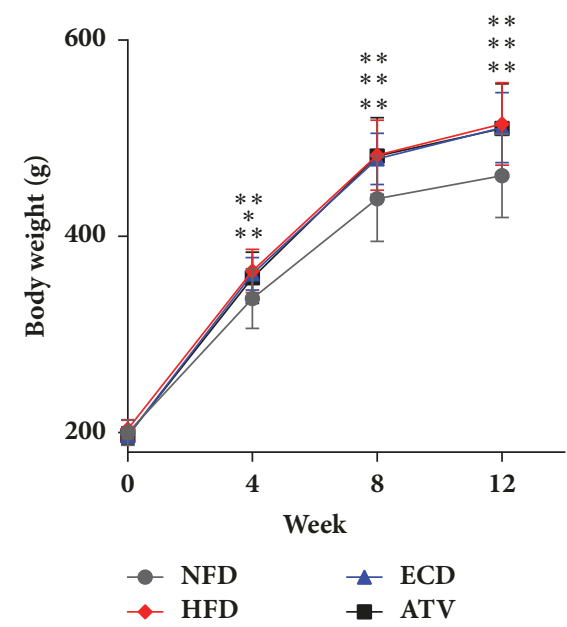

(a)

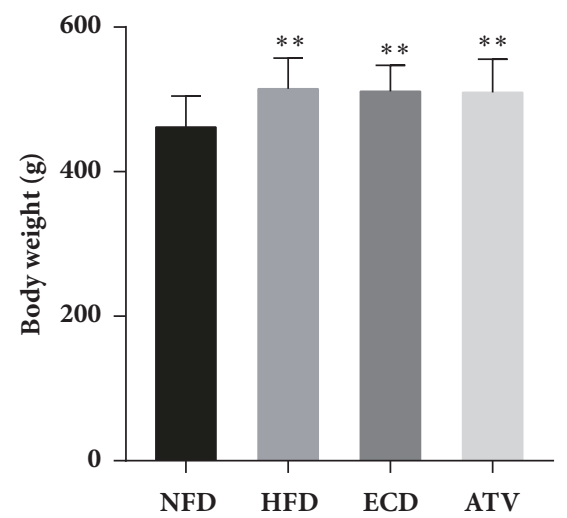

(c)

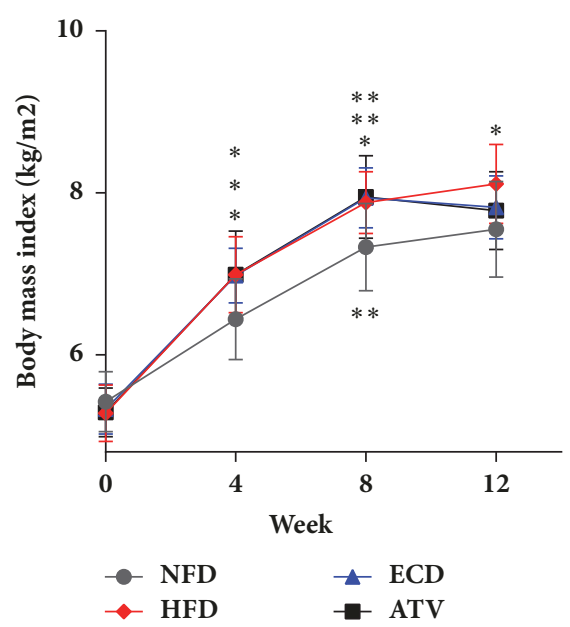

(b)

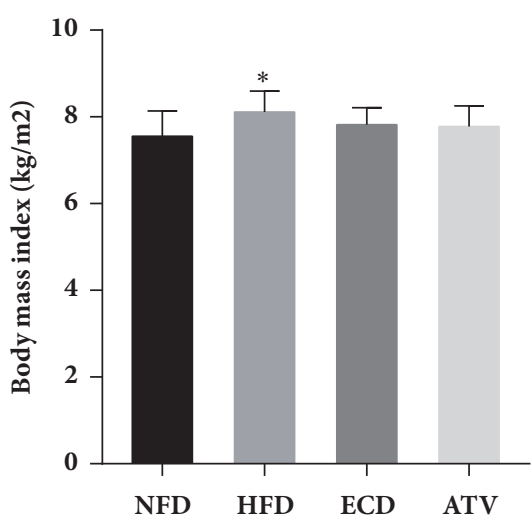

(d)

Figure 2: The changes of body weight and Body Mass Index in the NFD, HFD, ECD, and ATV groups. (a) Body weight and (b) Body Mass Index (weeks 0-4-8-12). (c) Body weight and (d) Body Mass Index (week 12). The data are presented as mean \pm SEM ( $\mathrm{n}=14), * p<0.05$ and $* * p<0.01$ versus NFD.

the TG and LDL-C levels, while they increased significantly the HDL-C and FFAs levels in animals fed HFD (Figure 4). The parameter TC was only significantly decreased following administration of ATV and not ECD compared with the HFD group (Figure 4).

In order to determine the success of model establishment, the change of blood lipid levels was measured and compared before ECD and ATV intervention. Significant elevations were noted for the indices TC during the $8^{\text {th }}$ week and not the $4^{\text {th }}$ week for the groups HFD, ECD, and ATV, whereas HDL$\mathrm{C}$ was significantly decreased in the aforementioned groups on the $8^{\text {th }}$ week (Supplementary Figure 1). The parameters TG and LDL-C were significantly increased on both the $4^{\text {th }}$ and the $8^{\text {th }}$ weeks for the groups HFD, ECD, and ATV (Supplementary Figure 1).

3.4. ECD Decreases the Levels of $L D L$ and oxLDL and Increases the Levels of HDL and CETP in HFD Rats. The serum concentrations of the lipoproteins HDL, VLDL, LDL, and oxLDL as well as the protein CETP were measured in the 4 groups examined. The HFD group exhibited significantly higher levels of LDL and oxLDL and significantly lower levels of HDL and CETP compared with these of the NFD group (Figures 5(a)-5(e)). Following intervention of HFD rats with ECD and ATV, the levels of LDL and oxLDL were significantly decreased, whereas the level of CETP was significantly increased (Figures 5(a)-5(e)). The level of HDL was only significantly increased following administration of ECD and not ATV compared with the HFD group. The level of VLDL did not significantly change in the 4 groups (Figure 5(a)).

3.5. The Effects of ECD on the Expression of Lipid Transport Factors in Liver and Visceral Fat of HFD Rats. To explore the underlying mechanisms of the regulation of ECD on the lipid metabolism disorder induced by HFD, the mRNA and protein expression levels of the protein CAV-1, the receptors VLDLR, LDLR, and the transporters ABCA1 and SRB1 were investigated in the liver and visceral adipose tissues. In the liver, CAV-1, LDLR, and ABCA1 mRNA expression levels were decreased significantly following HFD, while SRB1 mRNA expression levels were markedly increased 


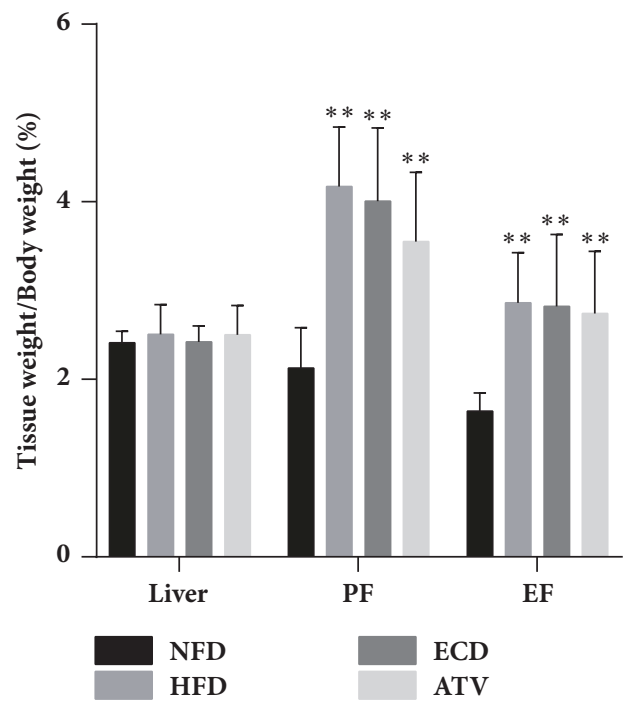

FIGURE 3: Measurements of percentage of liver, perirenal fat (PF), and epididymis fat (EF) tissue weight to body weight in the NFD, HFD, ECD, and ATV groups (week 12). The data are presented as mean \pm SEM $(\mathrm{n}=14), * p<0.05$ and $* * p<0.01$ versus NFD.

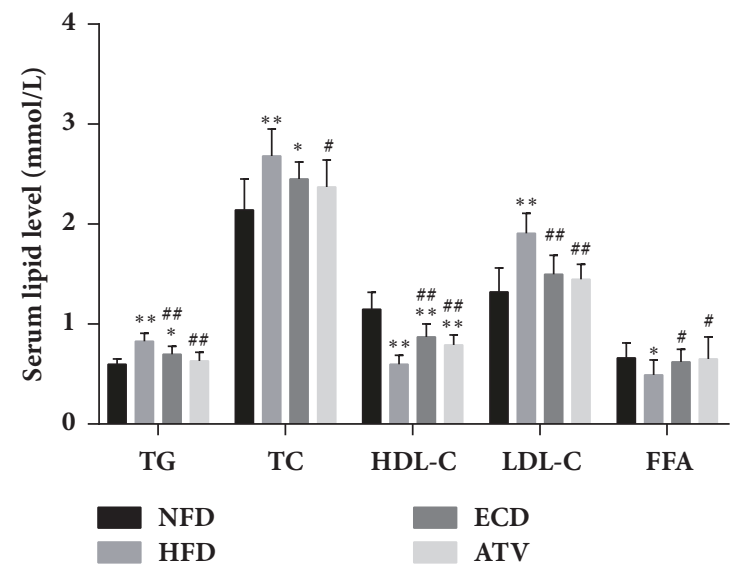

FIgURE 4: Biochemical measurements of serum lipid levels (TG, TC, HDL-C, LDL-C, and FFA) in the NFD, HFD, ECD, and ATV groups (week 12). The data are presented as mean \pm SEM $(n=14), * p<0.05$ and $* * p<0.01$ versus NFD; $\# p<0.05$ and $\# \# p<0.01$ versus HFD.

(Figure 6(a)). ECD and ATV treatment increased significantly the mRNA expression levels of CAV-1, LDLR, and ABCA1, whereas it significantly lowered the mRNA levels of SRB1 in HFD rats (Figure 6(a)). With regard to visceral fat, a similar pattern was noted for CAV-1, LDLR, and ABCA1 mRNA levels (Figure 6(b)). The mRNA levels of VLDLR were significantly increased in the HFD group compared with the NFD group and subsequently decreased in the ECD and ATV groups, although this change was not statistically significant (Figure 6(b)). SRB1 levels in visceral fat tissues were decreased significantly in the HFD rats and subsequently significantly increased in the ECD and ATV groups (Figure 6(b)). These results were confirmed by western blotting (Figures 6(c)-6(f)).

\section{Discussion}

According to TCM, the disorder of lipid metabolism is considered to be affected by the retention of phlegm dampness. HFD causes spleen and stomach impairment and leads to the accumulation of grease in the body, and finally the excess grease induces the retention of phlegm dampness [10]. The present study examined the lipid lowering effects of ECD in rats that were fed a high-fat diet. ECD was capable of reducing the levels of TC, TG, and LDL-C indices and further responsible for the increase in the levels of HDL-C in HFD rats, while it concomitantly did not affect considerably the food, water, and energy intake of HFD rats. These effects were comparable to the antiatherogenic agent atorvastatin (ATV). Additional investigation in the mechanism of action of the lipid lowering effect of ECD revealed that this extract could upregulate CAV-1, LDLR, and ABCA1 levels in the liver and visceral fat tissues and downregulate SRB1 levels in the liver while upregulate SRB1 levels in the visceral fat tissues of HFD animals. Taken collectively, the data suggested that ECD was an effective lipid lowering mixture of compounds that acted by regulating the apolipoprotein, apolipoprotein receptor, and lipid transport levels.

A limited number of studies have been conducted with regard to ECD and its applications for the treatment of various disorders. ECD has been reported to be used therapeutically for chronic bronchitis [42]. Furthermore, ECD ameliorated nonalcoholic steatohepatitis in a rat model of high-fat diet, as determined by reduced hepatic lipid disposition and liver injury and lower serum biochemistry markers, whereas in a similar study it was shown to improve insulin resistance and liver damage in rats, as evidenced by serum aminotransferase levels and histopathological examination [22, 43]. ECD is a basic herb for the treatment of drying dampness and resolving phlegm and has been widely reported to improve the disorder of lipid metabolism $[44,45]$. Animal experiments indicated that ECD could reduce blood glucose and regulate lipid metabolism [46], so as to ameliorate insulin resistance [23] or atherosclerosis [47]. In the present study, treatment with ECD significantly reduced the levels of TC, TG, and LDL-C and improved the levels of HDL-C and FFA in HFD rats. Furthermore, following HFD, ECD intervention decreased the levels of LDL and oxLDL and increased the levels of HDL and CETP, suggesting that ECD can effectively ameliorate disturbance of lipid metabolism induced by HFD in rats. A previous study conducted by Zhang and coworkers reported similar findings, and ECD was shown to improve TC and LDL$\mathrm{C}$ levels of early hyperlipidemia and atherosclerosis animal models [48]. These changes were evident on weeks 2 and 4 of the HFD animals [48]. The present study is in agreement with the aforementioned changes in the lipid parameters and the effects of ECD, although there were some differences between the studies, such as the animal model (lipid metabolism disorder model versus hyperlipidemia and atherosclerosis model), the dose $(4.35 \mathrm{~g} / \mathrm{kg}$ versus $4.8 \mathrm{~g} / \mathrm{kg})$, and the time point ( 8 weeks versus 5 days after duplicated the model) of administration. 


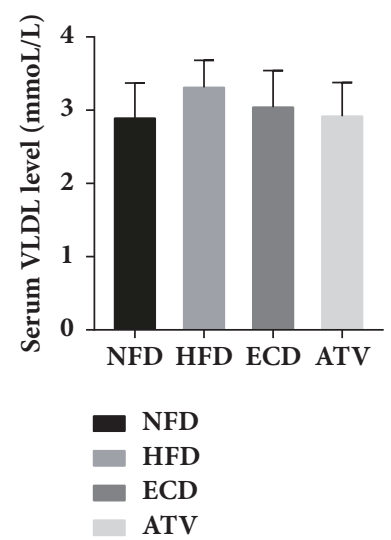

(a)

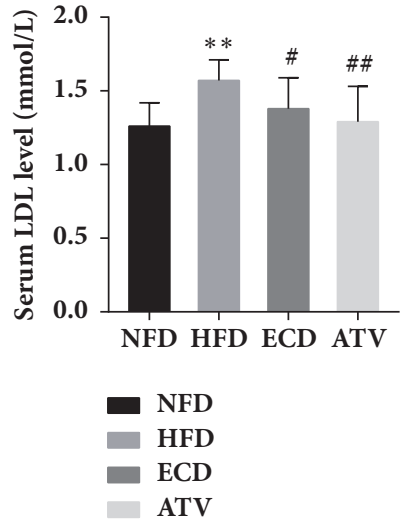

(b)

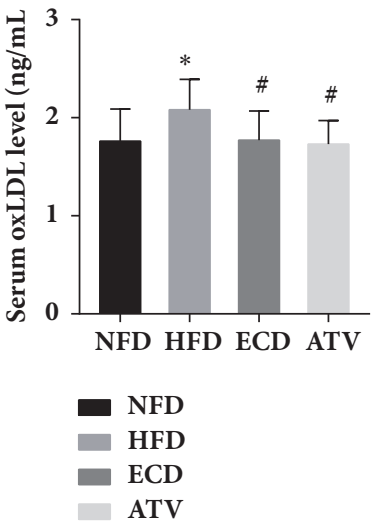

(c)

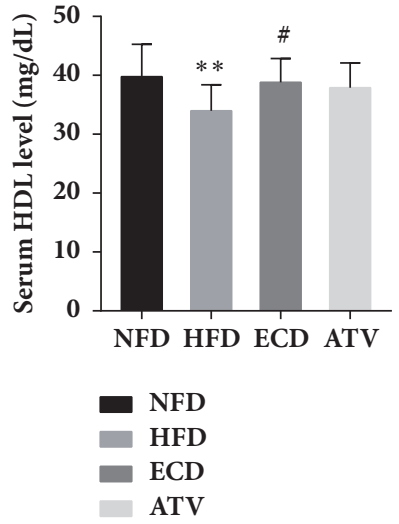

(d)

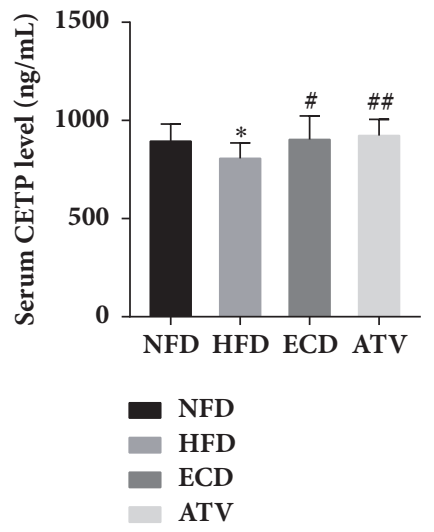

(e)

FIgURE 5: Measurement of the levels of lipoproteins and the cholesterol transfer protein CETP in the NFD, HFD, ECD, and ATV groups (week 12). (a) VLDL. (b)LDL. (c) oxLDL. (d) HDL. (e) CETP. The data are presented as mean \pm SEM ( $\mathrm{n}=14$ ), $* p<0.05$ and $* * p<0.01$ versus NFD; $\# p<0.05$ and $\# \# p<0.01$ versus HFD.

Based on the data presented in the current study, we hypothesized that the regulation of lipid transport was carried out in caveolae (Figure 7). These structures are considered the underlying factors required for ECD to alleviate dry dampness and resolved phlegm and ultimately to regulate lipid metabolism disorders. Caveolae is a site where lipids are transported intracellularly and extracellularly. CAV-1 is considered an intracellular lipid trafficking factor that can transport lipids and shuttles between the cytoplasm and the cell membrane via the caveolar vesicles $[49,50]$. Cholesterol efflux is increased in HepG2 cells transfected with a CAV1 expression plasmid DNA [51]. Moreover, it was confirmed that CAV-1 is a lipid-droplet protein [52-54] that can transfer lipids to lipid droplets for storage. In addition, VLDLR and LDLR can mediate the necessary lipoprotein/lipid transport into the cells, so as to avoid the accumulation of lipids in the blood [55-58], whereas ABCA1 can mediate cholesterol efflux. ABCA1 in adipocytes influences adipocyte lipid metabolism, body weight, and whole-body glucose homeostasis [59]. It has been shown that the expression of ABCA1 in macrophages from CAV-1 knockout mice is lower than that of the control group, indicating that CAV-1 is involved in regulating ABCA1-mediated cholesterol efflux
[60]. HDL presents or accepts cholesterol while anchored to plasma membranes via its receptor, SRB1. SRB1 mediates not only CE selective uptake (mainly in hepatocytes) but also cholesterol efflux (mainly in perithelial cells) [30]. It has been reported that caveolae present in cell membranes are the initial receiving sites of SRB1-mediated CE intake and $80 \%$ of CE accumulates in caveolae [61]. LDLR, SRB1 and ABCA1 are localized in caveolae [62].

To confirm this hypothesis, we measured the expression levels of the genes involved in the lipid transport in the rat liver and visceral fat tissues. HFD decreased the expression levels of CAV-1, LDLR, and ABCA1 and increased the expression levels of SRB1 in the liver tissues. Concomitantly, the expression levels of CAV-1, LDLR, ABCA1, and SRB1 were inhibited by HFD in the visceral fat, while the expression of VLDLR was increased. Following ECD intervention, the abnormal expression levels of these genes were reversed to their initial baseline values, indicating that the effect of ECD in the improvement of the disorder of lipid metabolism was mainly attributed to its ability to regulate the expression levels of lipid transporters in caveolae.

The mechanism by which ECD exerts lipid lowering effects in HFD animals remains unclear. Previous studies 


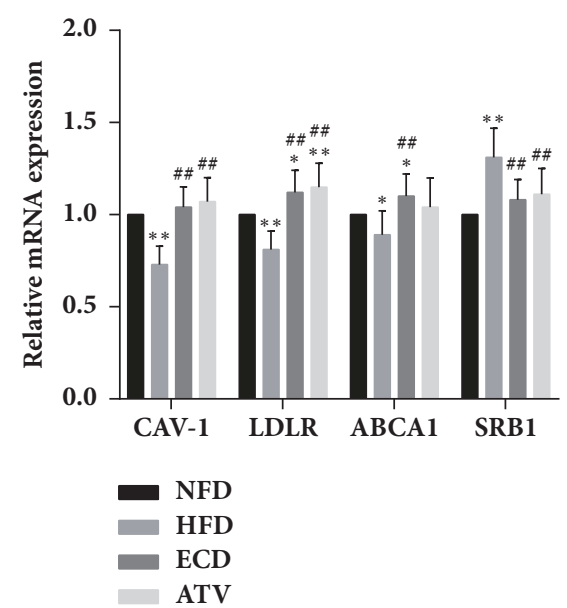

(a)

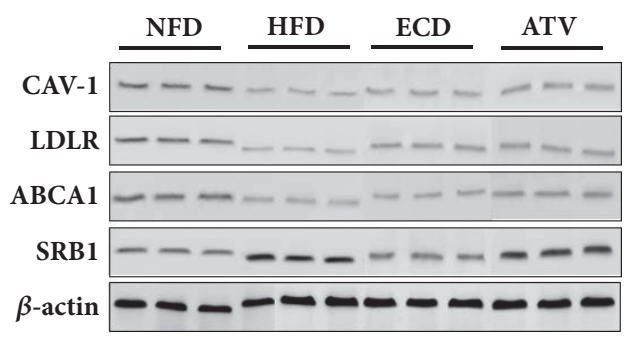

(c)

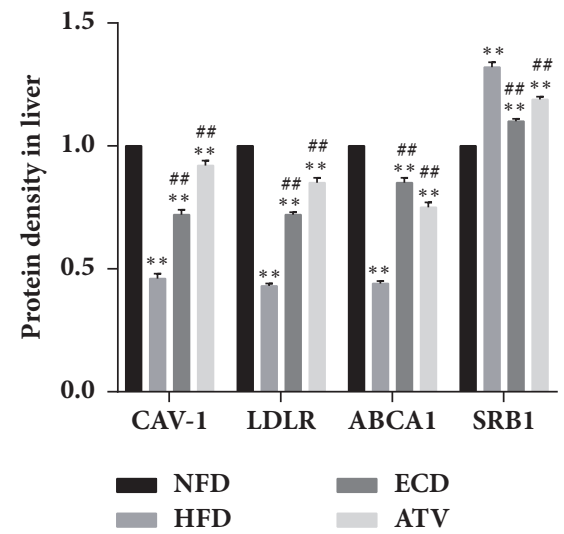

(e)

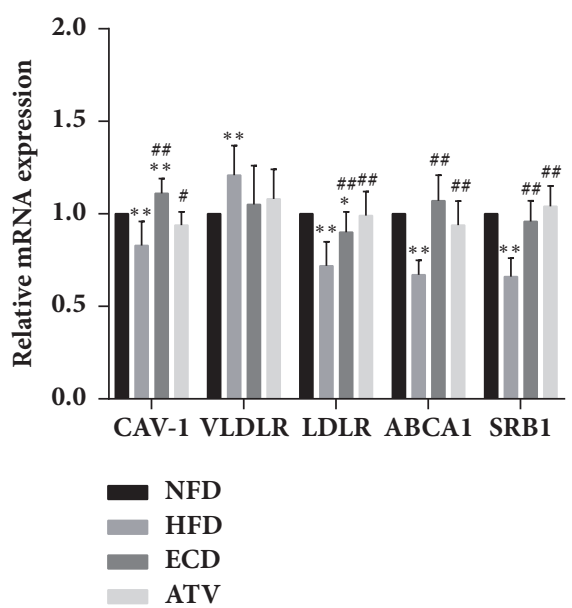

(b)

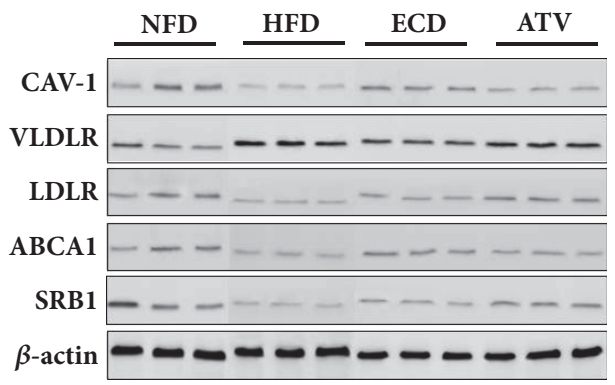

(d)

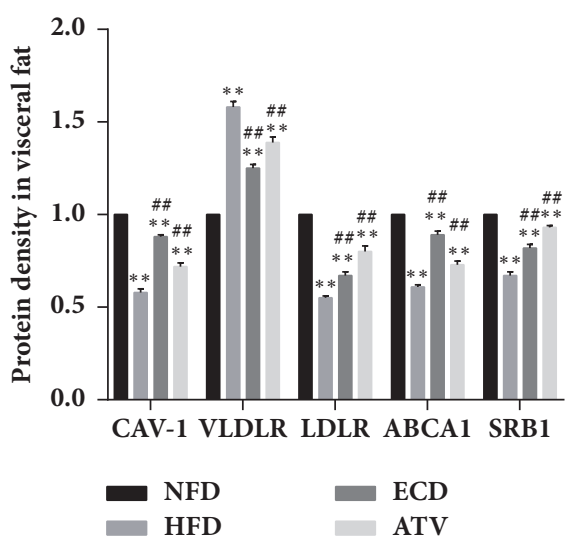

(f)

FIGURE 6: The mRNA and protein expression levels of lipid transporters, as determined by qPCR and Western immunoblotting: CAV-1, VLDLR, LDLR, ABCA1, and SRB1. (a) Liver tissue (mRNA); (b) visceral adipose tissue (mRNA). Western blotting of the expression levels of the proteins CAV-1, VLDLR, LDLR, ABCA1, and SRB1. (c) Liver tissue; (d) visceral adipose tissue. Semiquantification of the expression levels of the proteins examined with regard to the levels of $\beta$-actin. (e) Liver tissue. (f) Visceral adipose tissue. The data are presented as mean \pm SEM $(\mathrm{n}=14), * p<0.05$ and $* * p<0.01$ versus NFD; $\# p<0.05$ and $\# \# p<0.01$ versus HFD.

have shown that ECD could downregulate the MAPK JNK and the transcription factor AP-1, while it could further upregulate $\mathrm{p}-\mathrm{PKB}$ in HFD rats that had nonalcoholic steatohepatosis [22]. The study by Zhang et al. further demonstrated that ECD could decrease TNF- $\alpha$ and NF- $\kappa$ B levels in HFD-induced hyperlipidemic rats [43]. These studies have examined the mechanism of action of ECD with regard to cell signaling and inflammation, respectively. However the modulation of the expression levels of the lipid transporters CAV-1, LDLR, ABCA1, and SRB1 by ECD in the liver and visceral fat tissues of HFD animals has not been investigated to date. However, previous studies have shown that ATV treatment in rainbow trout, in vitro hepatocytes, and hyperlipidemic men can increase the levels of LDLR [6365]. Since ECD exhibited similar LDL-C and TC lowering effects, to those of ATV it is conceivable that a similar 


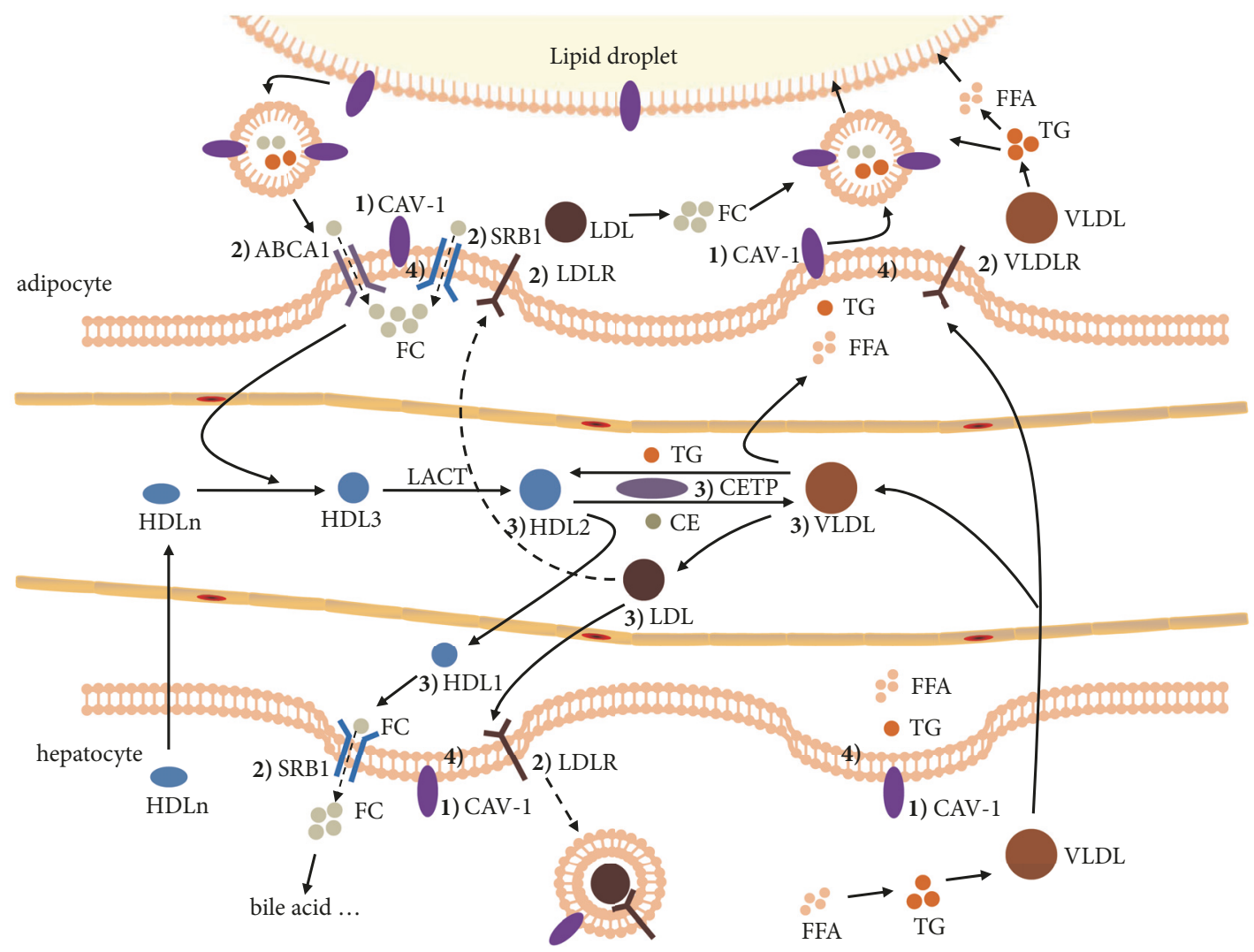

Figure 7: The model of lipid transport in caveolae. This model includes (1) an intracellular lipid trafficking system of CAV-1, (2) a transmembrane lipid transport system of lipoprotein receptors, (3) an extracellular lipid trafficking system of lipid transporters, and (4) a caveolae transport center.

pattern of regulation with regard to the protein CAV-1 and the receptor LDLR would be expected, as these markers are both involved in cholesterol metabolism. More importantly, ATV was reported to increase the levels of ABCA1 under HFD conditions in the liver and adipose tissues of hypertensive rats, which agrees with the present findings [66]. The present study adds further insight to the mechanism of lipid lowering effects mediated by ECD.

Long-term intake of high-fat diet induces a variety of reactions to cause lipid metabolism disorder in the body, such as insulin resistance (IR) [22, 43], inflammation [67], and endoplasmic reticulum (ER) stress [68]. These reactions will promote abnormal fat synthesis and decomposition through related factors and signaling pathways. Considering the key role of IR activation in regulating lipid metabolism, early studies have shown that ECD can regulate lipid metabolism by improving IR. Much data demonstrate a fundamental involvement of caveolae in insulin action [69]; this is why we studied the mechanism of ECD on regulating lipid metabolism from the perspective of caveolae. Now we are also exploring the effect of ECD on ER stress caused lipid metabolic disorders, and we will continue to investigate the molecular mechanisms and active constituents of ECD on regulating lipid metabolism in the future.

\section{Conclusions}

In summary, we have demonstrated that ECD exhibits a similar therapeutic effect on lipid metabolism disorder with that of ATV in HFD rats. Treatments with ECD ameliorated the lipid transport in caveolae. Further studies should be conducted to identify the effective constituents of ECD and molecular targets that are responsible for ameliorating the lipid metabolism disorder by ECD.

\section{Data Availability}

The data used to support the findings of this study are included within the article.

\section{Conflicts of Interest}

All authors declare that they have no any conflicts of interest.

\section{Acknowledgments}

This work was supported by National Natural Science Foundation (81503498) and Natural Science Foundation of Fujian Province (2016J01780, 2016J01398). 


\section{Supplementary Materials}

Supplementary Figure 1: biochemical measurements of serum lipid levels in the NFD, HFD, ECD, and ATV groups. (A) TG, (B) TC, (C) HDL-C, and (D) LDL-C (weeks 0-4-8). The data are presented as mean \pm SEM $(\mathrm{n}=14) ; * \mathrm{p}<0.05$ and $* * \mathrm{p}<0.01$ versus NFD. (Supplementary Materials)

\section{References}

[1] K. G. Parhofer, "The Treatment of Disorders of Lipid Metabolism," Deutsches Arzteblatt International, vol. 113, no. 15, pp. 261-268, 2016.

[2] C. Shaodong, Z. Haihong, L. Manting, L. Guohui, Z. Zhengxiao, and Z.Ym, "Research of influence and mechanism of combining exercise with diet control on a model of lipid metabolism rat induced by high fat diet," Lipids in Health and Disease, vol. 12, no. 1, article no. 21, 2013.

[3] L. Manting, Z. Haihong, L. Jing, C. Shaodong, and L. Yihua, "The model of rat lipid metabolism disorder induced by chronic stress accompanying high-fat-diet," Lipids in Health and Disease, vol. 10, article no. 153, 2011.

[4] S. M. Farrag, M. A. Hamzawy, M. El-Yamany, M. Saad, and N. Nassar, "Atorvastatin in nano-particulate formulation abates muscle and liver affliction when coalesced with coenzyme Q10 and/or vitamin E in hyperlipidemic rats," Life Sciences, vol. 203, pp. 129-140, 2018.

[5] T. J. Khan, A. Kuerban, S. S. Razvi et al., "In vivo evaluation of hypolipidemic and antioxidative effect of 'Ajwa' (Phoenix dactylifera L.) date seed-extract in high-fat diet-induced hyperlipidemic rat model," Biomedicine \& Pharmacotherapy, vol. 107, pp. 675-680, 2018.

[6] Y. Zhang, Z. Liu, and M. Zhou, "Therapeutic effects of fibroblast growth factor21 against atherosclerosis via the NFkappaB pathway," Mol Med Rep, vol. 17, no. 1, pp. 1453-1460, 2018.

[7] C. Baigent, L. Blackwell, and J. Emberson, "Efficacy and safety of more intensive lowering of LDL cholesterol: a meta-analysis of data from 170,000 participants in 26 randomised trials," The Lancet, vol. 376, no. 9753, pp. 1670-1681, 2010.

[8] B. Pashaie, R. Hobbenaghi, and H. Malekinejad, "Antiatherosclerotic effect of Cynodon dactylon extract on experimentally induced hypercholesterolemia in rats," Veterinary Research Forum, vol. 8, no. 3, pp. 185-193, 2017.

[9] D. K. Singh, N. Kumar, A. Sachan et al., "Hypolipidaemic effects of Gymnema sylvestre on high fat diet induced dyslipidaemia in wistar rats," Journal of Clinical and Diagnostic Research, vol. 11, no. 5, pp. FF01-FF05, 2017.

[10] J. Zhou, H. Wang, Y. Lv, Z. Wang, and W. Xiao, "Antiatherosclerotic effect of Longxuetongluo Capsule in high cholesterol diet induced atherosclerosis model rats," Biomedicine \& Pharmacotherapy, vol. 97, pp. 793-801, 2018.

[11] C. Zhang and B. Zhao, "Discussion on the relationship between hyperlipidemia and spleen deficiency," Journal of Hubei College of Traditional Chinese Medicine, vol. 5, no. 1, pp. 9-11, 2005.

[12] X. T. Yang, "My Opinion on Chinese Medicine Lipid - lowering Treatment," Chinese Journal of Basic Medicine in Traditional Chinese Medicine, vol. 18, no. 2, pp. 194-195, 198, 2012.

[13] L. Q. Jia, "Study on the Transfer of Lipstick and the Reverse Transport of Cholesterol from the Theory of Spleen Master Transport," Journal of Traditional Chinese Medicine, vol. 54, no. 20, pp. 1793-1795, 2013.
[14] J. Li and J. W. Lian, Pharmacology of Traditional Chinese Medical Formulae, Press of Traditional Chinese Medicine, Beijing, China, 2016.

[15] H. J. Yang, N. Yim, K. J. Lee et al., "Simultaneous determination of nine bioactive compounds in Yijin-tang via highperformance liquid chromatography and liquid chromatography-electrospray ionization-mass spectrometry," Integrative Medicine Research, vol. 5, no. 2, pp. 140-150, 2016.

[16] B.-Z. Gao, J.-C. Chen, L.-H. Liao, J.-Q. Xu, X.-F. Lin, and S.-S. Ding, "Erchen Decoction Prevents High-Fat Diet Induced Metabolic Disorders in C57BL/6 Mice," Evidence-Based Complementary and Alternative Medicine, vol. 2015, Article ID 501272, 9 pages, 2015.

[17] F. M. Pan and Z. Gui, "Clinical observation of Erchen decoction on treatment of 42 cases in nonalcoholic fatty liver disease," Journal of Yangtze University (Natural Science Edition) Medicine, vol. 7, no. 4, pp. 13-14, 2010.

[18] X. P. Hong, "Clinical observation of Erchen decoction on treatment of 60 cases in metabolic syndrome," Zhejiang Journal of Traditional Chinese Medicine, vol. 46, no. 5, p. 343, 2011.

[19] S. S. Ding, "Effect of Erchen decoction on blood fat and insulin resistance in rats fed a high-fat diet," Medical Innovation of China, vol. 11, no. 15, pp. 22-24, 2014.

[20] S. S. Ding, "Effect of Erchen decoction on insulin resistance and related gene expression in rats fed a high-fat diet," Guangming Journal of Chinese Medicine, vol. 29, no. 9, pp. 1833-1836, 2014.

[21] Z. Yao, L. Zhang, and G. Ji, "Efficacy of polyphenolic ingredients of Chinese herbs in treating dyslipidemia of metabolic syndromes," Journal of Chinese Integrative Medicine, vol. 12, no. 3, pp. 135-146, 2014.

[22] T.-h. Xie, J.-x. Li, T.-y. Mao et al., "An ErChen and YinChen Decoction Ameliorates High-Fat-Induced Nonalcoholic Steatohepatitis in Rats by Regulating JNK1 Signaling Pathway," Evidence-Based Complementary and Alternative Medicine, vol. 2017, Article ID 4603701, 14 pages, 2017.

[23] J. J. L. Williams and T. M. Palmer, "Cavin-1: caveolae-dependent signalling and cardiovascular disease," Biochemical Society Transactions, vol. 42, no. 2, pp. 284-288, 2014.

[24] P. G. Frank, M. W. Cheung, S. Pavlides, G. Llaverias, D. S. Park, and M. P. Lisanti, "Caveolin-1 and regulation of cellular cholesterol homeostasis," American Journal of Physiology-Heart and Circulatory Physiology, vol. 291, no. 2, pp. H677-H686, 2006.

[25] B. Razani, S. E. Woodman, and M. P. Lisanti, "Caveolae: from cell biology to animal physiology," Pharmacological Reviews, vol. 54, no. 3, pp. 431-467, 2002.

[26] H. H. Patel, F. Murray, and P. A. Insel, "Caveolae as organizers of pharmacologically relevant signal transduction molecules," Annual Review of Pharmacology and Toxicology, vol. 48, pp. 359-391, 2008.

[27] P. F. Pilch and L. Liu, "Fat caves: Caveolae, lipid trafficking and lipid metabolism in adipocytes," Trends in Endocrinology \& Metabolism, vol. 22, no. 8, pp. 318-324, 2011.

[28] C. J. Fielding and P. E. Fielding, "Caveolae and intracellular trafficking of cholesterol," Advanced Drug Delivery Reviews, vol. 49, no. 3, pp. 251-264, 2001.

[29] A. Bist, P. E. Fielding, and C. J. Fielding, "Two sterol regulatory element-like sequences mediate up-regulation of caveolin gene transcription in response to low density lipoprotein free cholesterol," Proceedings of the National Acadamy of Sciences of the United States of America, vol. 94, no. 20, pp. 10693-10698, 1997. 
[30] D. F. Liao and L. Qin, "Caveolae and Caveolin-1: A Possible Platform of Reverse Cholesterol Transport and Inflammation in Atherosclerosis," Chinese Journal of Arterioscler, vol. 20, no. 5, pp. 385-392, 2012.

[31] D.-X. Luo, D.-L. Cao, Y. Xiong, X.-H. Peng, and D.-F. Liao, "A novel model of cholesterol efflux from lipid-loaded cells," Acta Pharmacologica Sinica, vol. 31, no. 10, pp. 1243-1257, 2010.

[32] Y. H. Zhan, "Progress in Very Low Density Lipoprotein Receptor and Lipid Metabolism," Journal of Medical Research, vol. 41, no. 10, pp. 185-187, 2012.

[33] Y. Zhang, K. L. Ma, X. Z. Ruan, and B. C. Liu, "Dysregulation of the low-density lipoprotein receptor pathway is involved in lipid disorder-mediated organ injury," International Journal of Biological Sciences, vol. 12, no. 5, pp. 569-579, 2016.

[34] S. Wang and J. D. Smith, "ABCA1 and nascent HDL biogenesis," BioFactors, vol. 40, no. 6, pp. 547-554, 2014.

[35] G. A. Graf, S. V. Matveev, and E. J. Smart, "Class B scavenger receptors, caveolae and cholesterol homeostasis," Trends in Cardiovascular Medicine, vol. 9, no. 8, pp. 221-225, 1999.

[36] X. Q. Xiong, "Researching status on cholesteryl ester transfer protein," Chinese Journal of New Drugs, vol. 13, no. 10, pp. 874877, 2004.

[37] C. X. Chen, Pharmacology of Traditional Chinese Medicine, Shanghai Scientific and Technical Publishers, Shanghai, China, 2006.

[38] H. Tang, "Rat hyperlipidemia model set up using lipid mulsion," Journal of Xinjiang Medical University, vol. 33, no. 4, pp. 355-357, 2010.

[39] J. Chen, "Study on regulatory effect of Danshensu on lipid metabolism of hyperlipidemia rats," China Journal of Chinese MateriaMedica, vol. 40, no. 2, pp. 313-317, 2015.

[40] H. F. Zhang, "Effects of three kinds of dietary fiber on glucolipid metabolism and oxidative stress in lipid metabolism disorder rats," Journal of Southeast University (Med Sci Edi), vol. 35, no. 1, pp. 83-88, 2016.

[41] K. J. Livak and T. D. Schmittgen, "Analysis of relative gene expression data using real-time quantitative PCR and the 2 $\Delta \triangle \mathrm{CT}$ method," Methods, vol. 25, no. 4, pp. 402-408, 2001.

[42] L. P. Chen, Y. M. Cai, and J. S. Li, "Medication rules of famous veteran traditional Chinese medicine doctor in treatment of chronic bronchitis based on implicit structure model," Zhongguo Zhong Yao Za Zhi, vol. 42, no. 8, pp. 1609-1616, 2017.

[43] H. Zhang, N. Ta, P. Chen, and H. Wang, "Erchen decoction and linguizhugan decoction ameliorate hepatic insulin resistance by inhibiting IRS-1Ser307 phosphorylation in vivo and in vitro," Evidence-Based Complementary and Alternative Medicine, vol. 2017, Article ID 1589871, 11 pages, 2017.

[44] Z. Q. Chen, "Study on Erchen Decoction and Its Clinical Application," China Practical Medicine, vol. 8, no. 13, pp. 256257, 2013.

[45] F. T. Gao and S. S. Liu, "Clinical Observation on Erchen Decoction in Treating Hyperlipidemia," Guide of China Medicine, vol. 11, no. 5, pp. 287-288, 2013.

[46] T. Y. Wu, "Influence of Erchen Decoction on High-fat-diet Wistar Rats' Weight and Lipid Metabolism," Journal of Zhejiang University of TCM, vol. 36, no. 11, pp. 1218-1220+1238, 2012.

[47] X. J. Xie, "Effects of the Chinese medicine, modified Erchen decoction, on the lipid metabolism and hepatocyte morphology in ApoE-/-mice," Chinese Journal Of Comparative Medicine, vol. 25, no. 4, pp. 44-47, 2015.
[48] L. Zhang, Q. Zhang, Y. You et al., "Investigation of Evolution Rules of Phlegm and Blood Stasis Syndrome in Hyperlipidemia and Ath- erosclerosis by NMR-based Metabolic Profiling and Metabonomic Approaches," Zhongguo Zhong Xi Yi Jie He Za Zhi, vol. 35, no. 7, pp. 823-833, 2015.

[49] L. Qin and D. F. Liao, "Caveolae/caveolin- 1 and cellular cholesterol transport," Chinese Journal of Pathophysiology, vol. 23, no. 10, pp. 2067-2070, 2007.

[50] H. N. Fridolfsson, D. M. Roth, P. A. Insel, and H. H. Patel, "Regulation of intracellular signaling and function by caveolin," The FASEB Journal, vol. 28, no. 9, pp. 3823-3831, 2014.

[51] C. Fu, J. He, C. Li, J. Y.-J. Shyy, and Y. Zhu, "Cholesterol increases adhesion of monocytes to endothelium by moving adhesion molecules out of caveolae," Biochimica et Biophysica Acta (BBA) - Molecular and Cell Biology of Lipids, vol. 1801, no. 7, pp. 702$710,2010$.

[52] D. L. Brasaemle, G. Dolios, L. Shapiro, and R. Wang, "Proteomic analysis of proteins associated with lipid droplets of basal and lipolytically stimulated 3T3-L1 adipocytes," The Journal of Biological Chemistry, vol. 279, no. 45, pp. 46835-46842, 2004.

[53] S. Le Lay, E. Hajduch, M. R. Lindsay et al., "Cholesterol-induced caveolin targeting to lipid droplets in adipocytes: A role for caveolar endocytosis," Traffic, vol. 7, no. 5, pp. 549-561, 2006.

[54] A. Öst, U. Örtegren, J. Gustavsson, F. H. Nystrom, and P. Strålfors, "Triacylglycerol is synthesized in a specific subclass of caveolae in primary adipocytes," The Journal of Biological Chemistry, vol. 280, no. 1, pp. 5-8, 2005.

[55] T. T. Sun, "Expression and distribution of VLDLR and its subtypes in various tissues of type 2 diabetic rat," Chinese Journal of Diabetes, vol. 16, no. 5, pp. 304-306, 2008.

[56] T. T. Sun, "Effect of Insulin on VLDLR Gene Expression in 3T3L1 Adipocyte," Chinese Journal of Biochemistry and Molecular Biology, vol. 23, no. 6, pp. 457-462, 2007.

[57] X. Q. Luo, "Effect of total flavones of CrataegiFructus on lipid metabolism and expression of low density lipoprotein receptor in rats with fatty liver induced by complex factors," ChineseTraditional and Herbal Drugs, vol. 42, no. 7, pp. 13671373, 2011.

[58] C. Leeb, C. Eresheim, and J. Nimpf, "Clusterin is a ligand for apolipoprotein e receptor 2 (ApoER2) and very low density lipoprotein receptor (VLDLR) and signals via the reelinsignaling pathway," The Journal of Biological Chemistry, vol. 289, no. 7, pp. 4161-4172, 2014.

[59] W. de Haan, A. Bhattacharjee, P. Ruddle, M. H. Kang, and M. R. Hayden, "ABCA1 in adipocytes regulates adipose tissue lipid content, glucose tolerance, and insulin sensitivity," Journal of Lipid Research, vol. 55, no. 3, pp. 516-523, 2014.

[60] Y.-C. Lin, C. Ma, W.-C. Hsu, H.-F. Lo, and V. C. Yang, "Molecular interaction between caveolin-1 and ABCA1 on high-density lipoprotein-mediated cholesterol efflux in aortic endothelial cells," Cardiovascular Research, vol. 75, no. 3, pp. 575-583, 2007.

[61] J. V. Mulcahy, D. R. Riddell, and J. S. Owen, "Human scavenger receptor class B type II (SR-BII) and cellular cholesterol efflux," Biochemical Journal, vol. 377, no. 3, pp. 741-747, 2004.

[62] L. Qin, N. Zhu, B. X. Ao et al., "Caveolae and Caveolin-1 Integrate Reverse Cholesterol Transport and Inflammation in Atherosclerosis," International Journal of Molecular Sciences, vol. 17, no. 3, p. 429, 2016.

[63] A. A. Al-Habsi, "Atorvastatin alters gene expression and cholesterol synthesis in primary rainbow trout (Oncorhychus) hepatocytes," Comp BiochemPhysiol B BiochemMolBiol in Press, 2017. 
[64] Q. M. Melendez, C. J. Wooten, and D. Lopez, "Atorvastatin and lovastatin, but not pravastatin, increased cellular complex formation between PCSK9 and the LDL receptor in human hepatocyte-like C3A cells," Biochemical and Biophysical Research Communications, vol. 492, no. 1, pp. 103-108, 2017.

[65] A. J. Tremblay, B. Lamarche, V. Lemelin et al., "Atorvastatin increases intestinal expression of NPC1L1 in hyperlipidemic men," Journal of Lipid Research, vol. 52, no. 3, pp. 558-565, 2011.

[66] K. Akiyama, Y.-Q. Liang, M. Isono, and N. Kato, "Investigation of functional genes at homologous loci identified based on genome-wide association studies of blood lipids via high-fat diet intervention in rats using an in vivo approach," Journal of Atherosclerosis and Thrombosis, vol. 22, no. 5, pp. 455-480, 2015.

[67] Y. Z. Gong, S. W. Sun, and D. F. Liao, "Interaction and regulation of cell inflammation and lipid metabolism," Chinese Journal of Arterioscler, vol. 25, no. 6, pp. 623-629, 2017.

[68] T. Mao, L. Yang, and Y. Liu, "The mechanism of cell endoplasmic reticulum stress and glucose and lipid metabolism disorder," Chinese Journal of Cell Biology, vol. 33, no. 7, pp. 727-737, 2011.

[69] P. Stralfors, "Caveolins and caveolae, roles in insulin signalling and diabetes," Advanced Drug Delivery Reviews, vol. 49, no. 3, pp. 251-264, 2001. 


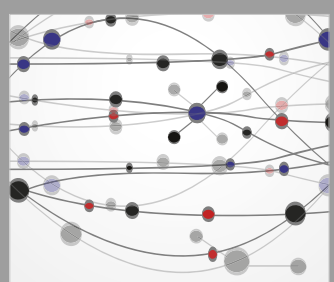

The Scientific World Journal
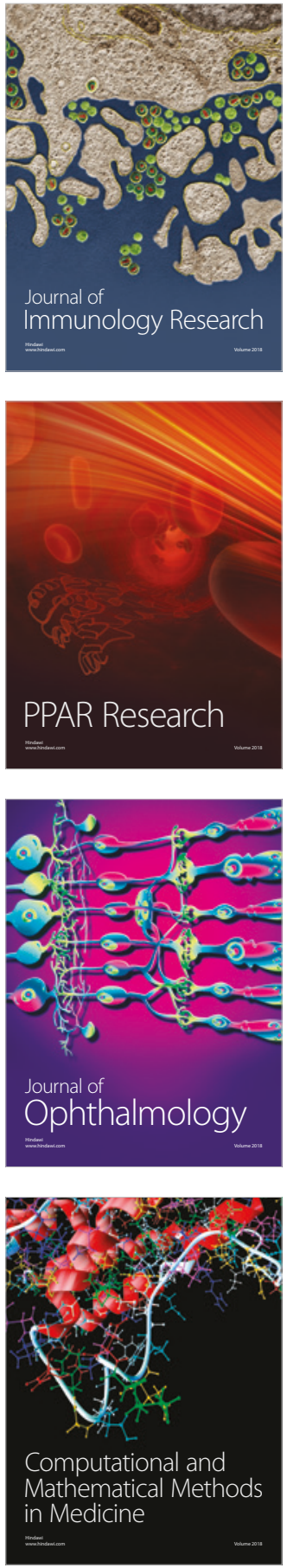

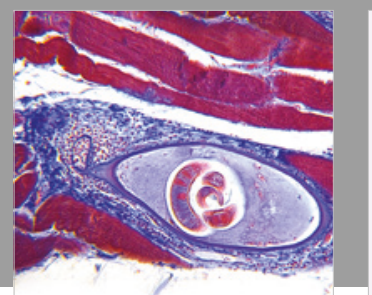

Gastroenterology Research and Practice

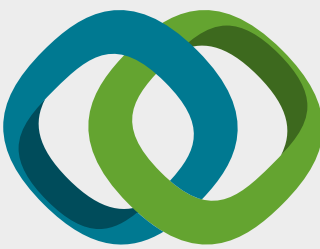

\section{Hindawi}

Submit your manuscripts at

www.hindawi.com
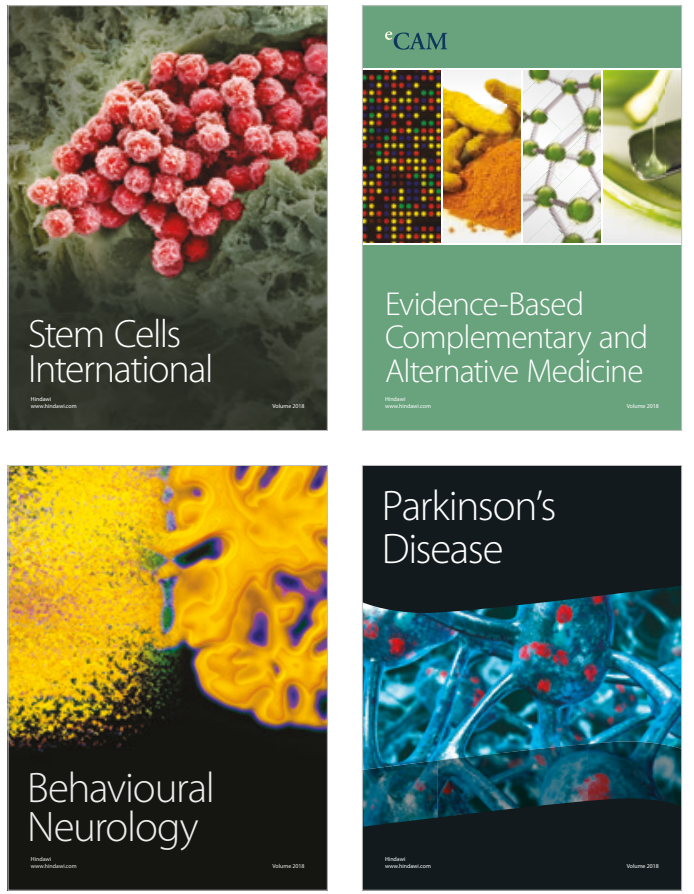

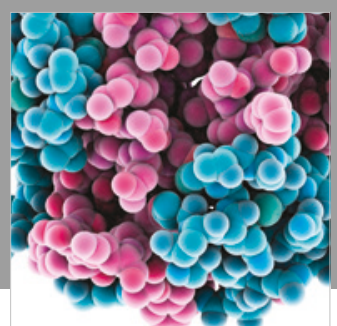

ournal of

Diabetes Research

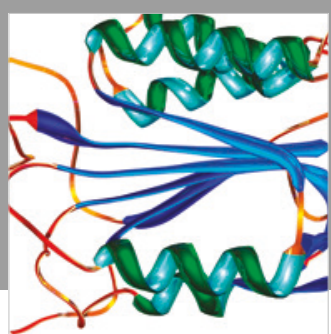

Disease Markers
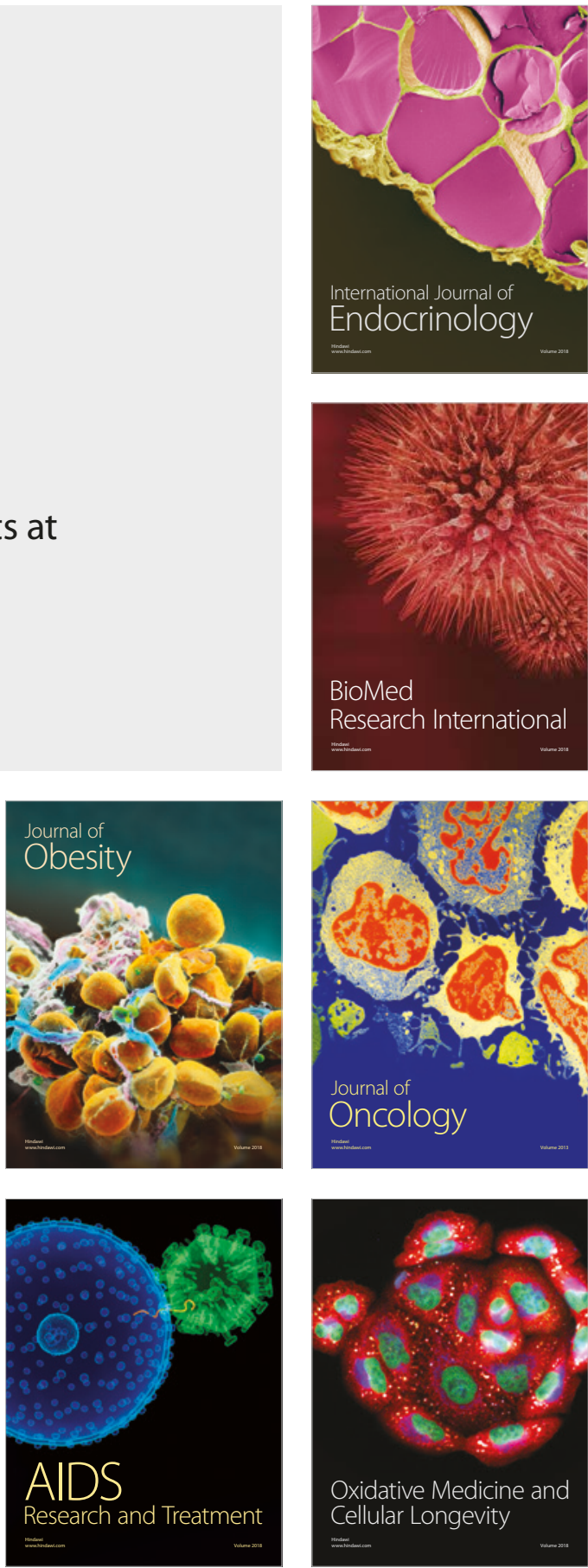